\title{
CALCULATION OF POSITION AND VELOCITY OF GLONASS SATELLITE BASED ON ANALYTICAL THEORY OF MOTION
}

\author{
W. Góral, B. Skorupa \\ AGH University of Science and Technology, Department of Geomatics, \\ al. A. Mickiewicza 30, 30-059 Krakow, Poland \\ e-mails: wgik@agh.edu.pl,bskorupa@agh.edu.pl
}

\begin{abstract}
The presented algorithms of computation of orbital elements and positions of GLONASS satellites are based on the asymmetric variant of the generalized problem of two fixed centers. The analytical algorithm embraces the disturbing acceleration due to the second $J_{2}$ and third $J_{3}$ coefficients, and partially fourth zonal harmonics in the expansion of the Earth's gravitational potential. Other main disturbing accelerations - due to the Moon and the Sun attraction - are also computed analytically, where the geocentric position vector of the Moon and the Sun are obtained by evaluating known analytical expressions for their motion. The given numerical examples show that the proposed analytical method for computation of position and velocity of GLONASS satellites can be an interesting alternative for presently used numerical methods.
\end{abstract}

Keywords: GLONASS, Eulerian orbit, Keplerian orbit, problem of two fixed centers, lunar and solar gravitational perturbations.

\section{INTRODUCTION}

According to GLONASS news section (http:|www.glonass-ianc.rsa.ru/en/) on 8th December 2011 the GLONASS constellation has reached the number of 24 operating satellites, providing $100 \%$ coverage of the Earth's surface. The availability of GLONASS satellites has brought two significant benefits to geodetic applications of global positioning systems. Firstly, the solution from GLONASS could be employed as an independent verification of GPS solution to improve the quality control. Secondly, GPS and GLONASS observations could be combined directly, and as a result the geometry of observed satellites could be enhanced by increasing the number of available satellites.

The GPS broadcast ephemerides employ a set of Kepler-like parameters to determine the satellite position. Therefore the algorithm of computing the GPS satellite position is completely different. The GPS broadcast ephemerides are updated hourly and are nominally valid for two hours either side of their specified reference time. In presented paper motion of the GLONASS satellite is described using osculating elements of the intermediate, nonKeplerian (Eulerian) orbit. This intermediate Eulerian orbit corresponds to the exact solution of the generalized problem of two fixed centers. Taking into account that L. Euler was the first who investigated the problem of two fixed centers and reduced it to quadratures, E.P Aksenov (1977) suggested in that case referring to it as Eulerian motion. Elements of the intermediate orbit are called Eulerian elements. Such a choice of orbital elements allows us to include the most significant perturbing forces into the satellite's intermediate motion. The 
intermediate orbit of the satellite is based on solving the generalized problem of two fixed centers which fully takes into account the influence of the second $J_{2}$ and third $J_{3}$ zonal, and a major part of the fourth zonal harmonic of the expansion of the Earth's gravity potential.

The equations of motion of the satellites in the generalized problem of two fixed centers are integrable in quadratures. After inversion of quadratures the Cartesian coordinates of a satellite are related to orbital parameters and time by transcendental relationships, which include Jacobi's elliptic functions. So, to simplify calculations E.P. Aksenov (1977) has made expansions of all obtained relations into series in terms of powers of two small parameters $\varepsilon^{2}$ and $\varepsilon \sigma$, where $\varepsilon=c / p, p=a\left(1-e^{2}\right)$ is semi-parameter of the orbit, $a$ is semi-major axis and $e$ is eccentricity of the non-Keplerian (Eulerian) intermediate orbit. For near-Earth's satellites $\varepsilon<1 / 30$. For GPS and GLONASS satellites this parameter is less than $1 / 100$. This made it possible to reduce the formulae of the theory of Eulerian motion to trigonometric form. The parameters of motion similar to Keplerian elements were taken as arbitrary constants of integration. The elegance of derived formulae is emphasized by the fact of their precise inversion into formulae of Keplerian motion, if small parameters $\varepsilon, \sigma$, are let to be zero. E. P. Aksenov (1977) derived all formulae of the theory to an accuracy of order $\varepsilon^{4}$ and $\sigma^{4}$. Various methods of integration of the equation of satellite motion are described in V.G. Demin's monograph (Demin, 1970), and E.P. Aksenov's monograph (Aksenov, 1977).

\section{FORMULATION OF THE PROBLEM}

Positioning of GLONASS satellites based on broadcast ephemerides differs in several aspects from that of GPS. The GLONASS broadcast ephemerides provide initial conditions every 30 minutes. They contain coordinates and velocities of the satellite (state vector) in Earthcentered, Earth-fixed ECEF (PZ-90.02) coordinate frame and the lunar-solar accelerations. According to GLONASS Interface Control Document (ICD 2008), in order to obtain satellite coordinates at a time different from that reference time, the satellite's equations of motion have to be numerically integrated. The GLONASS-ICD force model is recommended for only a time period of 15 minutes either side of the broadcast ephemerides reference time. A full description of the format and information contained in the GLONASS navigation message can be found in (GLONASS-ICD, 2008).

Let $O x y z$ be a rectangular geocentric coordinate system in which the origin falls at the center of mass of the Earth. The principal axis $x$ points towards the vernal equinox. The $x y$ plane coincides with the equatorial plane, and the $O z$ axis is directed along the Earth's rotation axis. The differential equations of motion for the GNSS satellite may be written in the form (Aksenov et al., 1963).

$$
\frac{d^{2} x}{d t^{2}}=\frac{\partial U}{\partial x}, \quad \frac{d^{2} y}{d t^{2}}=\frac{\partial U}{\partial y}, \quad \frac{d^{2} z}{d t^{2}}=\frac{\partial U}{\partial z},
$$

where the function $U$ is given by the equation

$$
U=\frac{G M}{2}\left\{\frac{1+i \sigma}{r_{1}}+\frac{1-i \sigma}{r_{2}}\right\},
$$

in which

$$
r_{1}=\sqrt{x^{2}+y^{2}+[z-c(\sigma+i)]^{2}}, \quad r_{2}=\sqrt{x^{2}+y^{2}+[z-c(\sigma-i)]^{2}},
$$

$G$ is the gravitational constant, $M$ is the mass of the Earth, and $i=\sqrt{-1}$.

The constants $c$ and $\sigma$ are strictly associated with the $J_{2}$ and $J_{3}$ (Aksenov et al., 1963) 


$$
c=a_{e} \sqrt{J_{2}-\left(\frac{J_{3}}{2 J_{2}}\right)^{2}}, \quad \sigma=\frac{J_{3}}{2 J_{2}} \cdot \frac{a_{e}}{c} .
$$

where $a_{\mathrm{e}}$ is the equatorial radius of the reference ellipsoid.

The function $U$ possesses two important properties. First, with an appropriate selection of $c$ an $\sigma$ it provides a good approximation to the gravitational potential of the Earth. The function $U$ will differ from the gravitational potential by terms of the second order in the flattening. Secondly, the equations of motion with the force function $U$ can be integrated in quadratures. These two properties enable us to construct intermediate orbits for the satellite that will be closer to true orbits than unperturbed Keplerian orbits would be (Aksenov et al., 1963).

\section{A COMPARISON OF THE EULERIAN AND KEPLERIAN ORBIT ELEMENTS ON THE EXAMPLE OF GLONASS SATELLITE}

Six independent parameters are required to describe the motion of a satellite around the Earth. The non-Keplerian (Eulerian) intermediate satellite orbit in the generalized problem of two fixed centers can simply be described by the following classical elements: $a$ is the semi-major axis, $e$ is eccentricity, $i(s=\sin i)$ is inclination, $\omega_{0}$ is the argument of perigee, $\Omega_{0}$ is the longitude of ascending node and $M_{0}$ is a mean anomaly at the epoch $t_{0}$. Eulerian elements are very useful for visualizing a given orbit in space. The above parameters are reduced to corresponding Keplerian elements, for $c=0$ and $\sigma=0$ (Aksenov, 1977). Knowing from broadcast ephemerides the geocentric position and velocity vectors $\mathbf{r}(t), \mathbf{v}(t)$, and using the formulae of the generalized problem of two fixed centers we can obtain to each epoch $t$ osculating Eulerian (and in the case $c=0, \sigma=0$, also Keplerian) elements: $a(t), e(t), i(t)$, $\omega_{0}(t), \Omega_{0}(t), M_{0}(t)$ (Aksenov, 1977). An example of formulae for calculation of intermediate orbital elements and computation of the GLONASS satellite position is provided step by step in our previous paper (Góral and Skorupa, 2012). Given the six elements, it is always possible to uniquely calculate the position and velocity vector of the satellite.

The following figures (Fig. 1a, 2a, 3a, 4a, 5a) show the osculating Eulerian elements as a function of time: $a(t), e(t), i(t), \Omega(t), \omega(t)$ over a time interval of one day for GLONASS satellite GLN 15, calculated at 48 time epochs from $0^{\mathrm{h}} 15^{\mathrm{m}}$ to $23^{\mathrm{h}} 45^{\mathrm{m}}$ (2015-01-13). For comparison in figures $1 \mathrm{~b}, \ldots, 5 \mathrm{~b}$, the same Keplerian orbital elements are presented. Additionally, in Fig. 6a and Fig. 6b, respectively the osculating Eulerian and Keplerian mean motion are given.

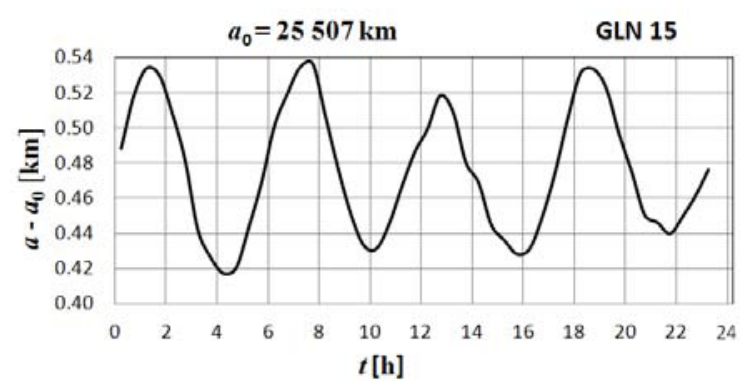

Fig. 1a. Osculating Eulerian semi-major axis of the satellite GLN 15 during one day (2015-01-13).

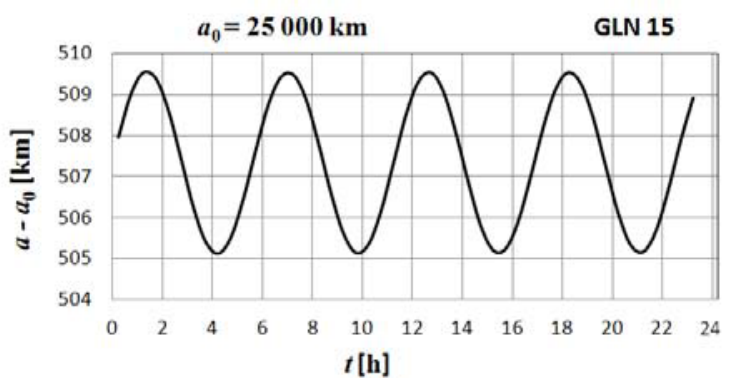

Fig. 1b. Osculating Keplerian semi-major axis of the satellite GLN 15 during one day (2015-01-13). 


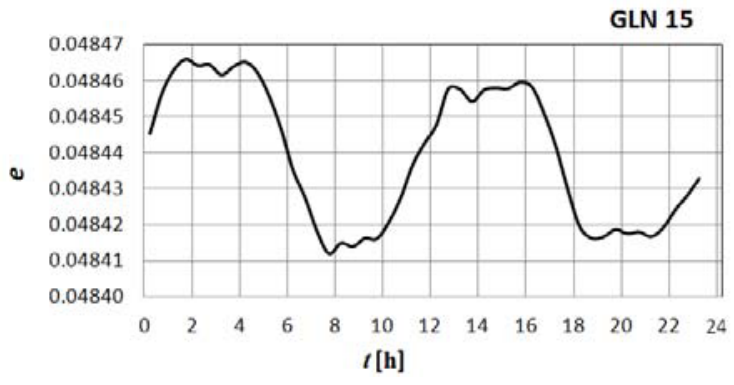

Fig. 2a. Osculating Eulerian eccentricity ot the satellite GLN 15 during one day (2015-01-13).

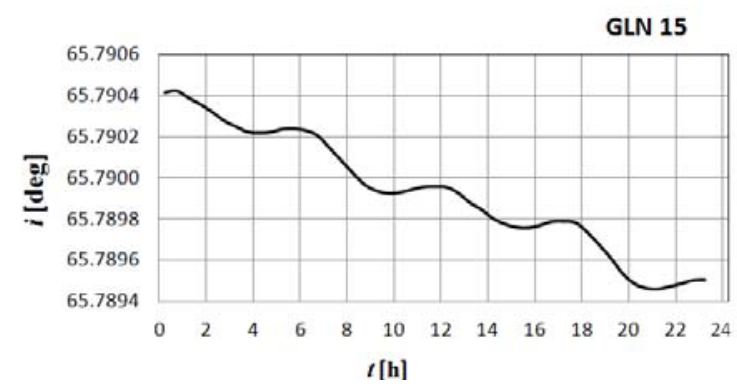

Fig. 3a. Osculating Eulerian inclination of the satellite GLN 15 during one day (2015-01-13).

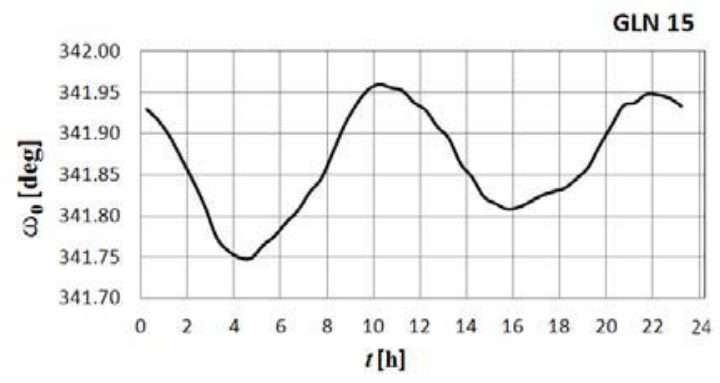

Fig. 4a. Osculating Eulerian argument of perigee of the satellite GLN 15 during one day (2015-01-13).

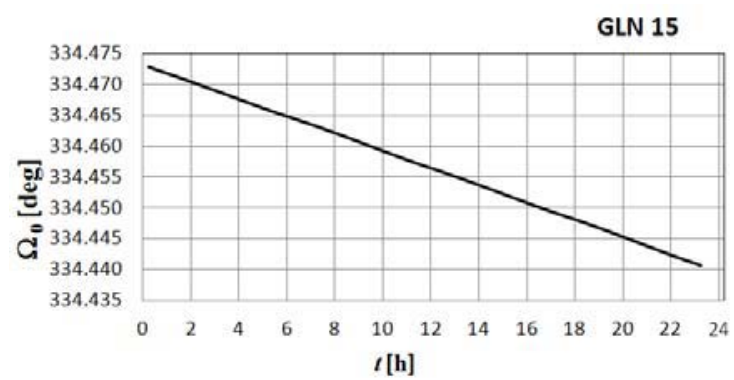

Fig. 5a. Osculating Eulerian longitude of ascending node of the satellite GLN 15 during one day (2015-01-13).

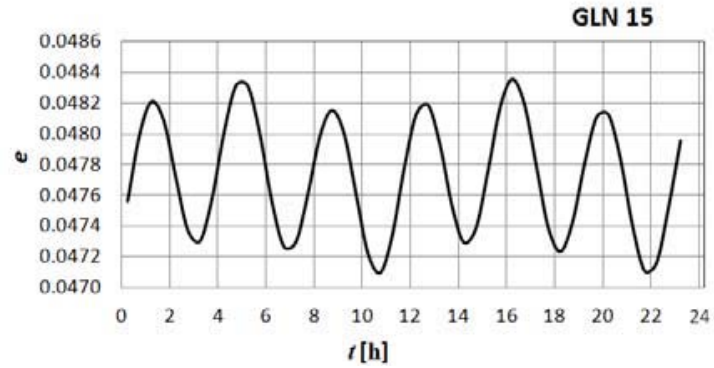

Fig. 2b. Osculating Keplerian eccentricity of the satellite GLN 15 during one day (2015-01-13).

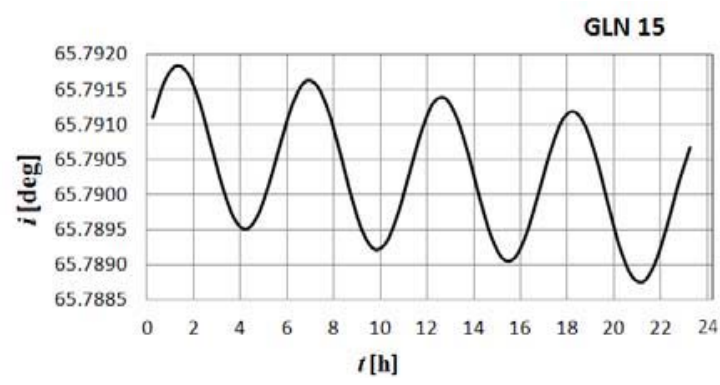

Fig. 3b. Osculating Keplerian inclination of the satellite GLN 15 during one day (2015-01-13).

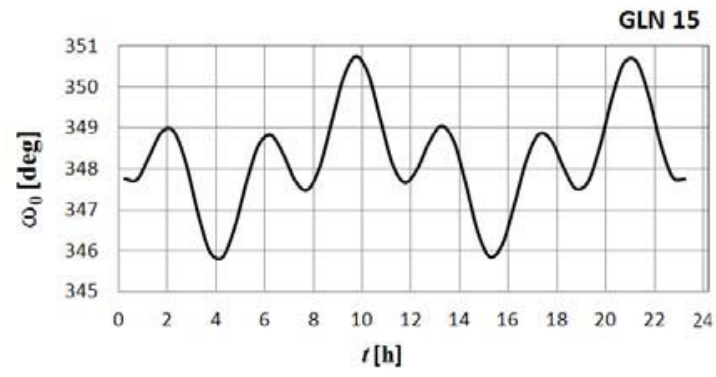

Fig. 4b. Osculating Keplerian argument of perigee of the satellite GLN 15 during one day (2015-01-13).

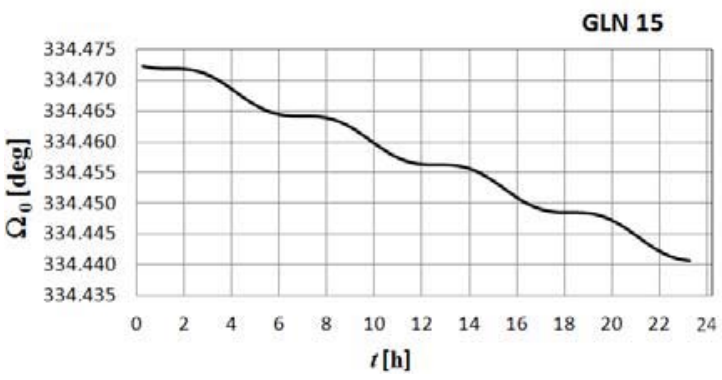

Fig. 5b. Osculating Keplerian longitude of ascending node of the satellite GLN 15 during one day (2015-01-13). 


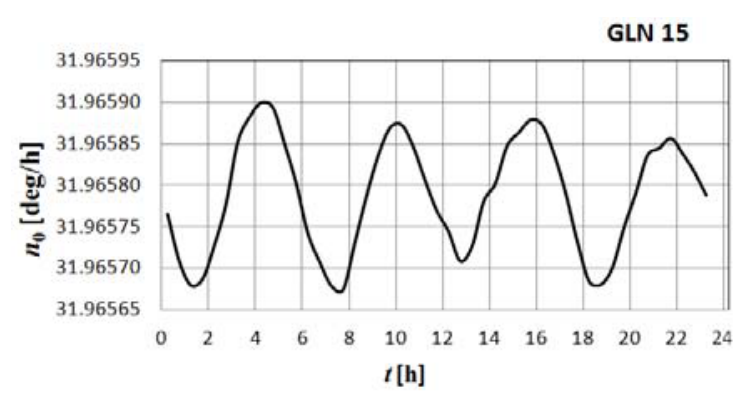

Fig. 6a. Osculating Eulerian mean motion of the satellite GLN 15 during one day (2015-01-13).

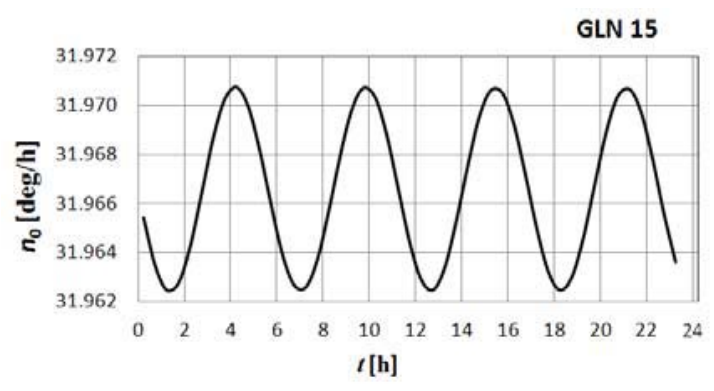

Fig. 6b. Osculating Keplerian mean motion of the satellite GLN 15 during one day (2015-01-13).

In the Keplerian elements $a(t), e(t), i(t), \omega(t)$ we see very pronounced short-period perturbations with the period of one satellite revolution or smaller, most of them caused by the Earth's oblateness. In Eulerian elements $a(t), e(t), i(t), \omega(t), \Omega(t)$ we see the result of removing effects caused by the Earth's oblateness. Moreover, one can see that the amplitude of Eulerian osculating element $a(t)$ is about twenty times smaller than one for Keplerian element and about ten (for $e(t)$ and $i(t)$ ) times smaller than one for Keplerian osculating elements.

One example of results of computation Eulerian and Keplerian elements at the epoch $t_{0}=12^{\mathrm{h}}$ $45^{\mathrm{m}} 00.0^{\mathrm{s}}$ UTC are presented in Table 1.

Table 1. Orbital elements of the GLONASS satellite GLN15 at epoch $t_{0}=12^{\mathrm{h}} 45^{\mathrm{m}} 00.0^{\mathrm{s}}$ UTC

\begin{tabular}{|c|c|r|r|}
\hline Item & Words [units] & Eulerian elements & Keplerian elements \\
\hline 1 & $\boldsymbol{a}[\mathrm{km}]$ & 25507.518483 & 25509.534901 \\
\hline 2 & $\boldsymbol{e}$ & 0.048457897602 & 0.0481908815 \\
\hline 3 & $\boldsymbol{i}[\mathrm{deg}]$ & 65.789924999 & 65.79138160 \\
\hline 4 & $\omega_{0}[\mathrm{deg}]$ & 341.90868955 & 348.64651746 \\
\hline 5 & $\Omega_{0}[\mathrm{deg}]$ & 334.45700346 & 334.45626261 \\
\hline 5 & $M_{0}[\mathrm{deg}]$ & 20.98443758 & 14.24681024 \\
\hline
\end{tabular}

One can see quite a difference between the Eulerian and Keplerian angular elements $\omega_{0}$ and $M_{0}$, while their sum $\omega_{0}+M_{0}$ does not differ that much.

\section{IMPACT OF THE LUNAR-SOLAR PERTURBATION ON THE POSITION AND VELOCITY OF THE GLONASS SATELLITE}

In presented paper the solution of the generalized problem of two fixed centers is taken as the undisturbed intermediate orbit. In the theory of satellite motions based on the generalized problem of two fixed centers the disturbance of the Eulerian orbit is caused primarily by the gravitational attraction of the Moon and the Sun. The calculation of the total lunar-solar accelerations were obtained according to the formulae given in GLONASS ICD 2008.

The position of the Sun was calculated with the help of Fortran library SOFA (Standards of Fundamental Astronomy) designed by International Astronomical Union and the position of the Moon was calculated with the help of simplified algorithm based on the paper (Van Flandern and Pulkkinen, 1979). Calculated acceleration components of the GLONASS GLN 15 due to lunar gravitational perturbation are presented in Fig. 7a, and due to solar 
gravitational perturbation are presented in Fig. 7b. These lunar and solar accelerations are related to the PZ-90.02 reference frame.

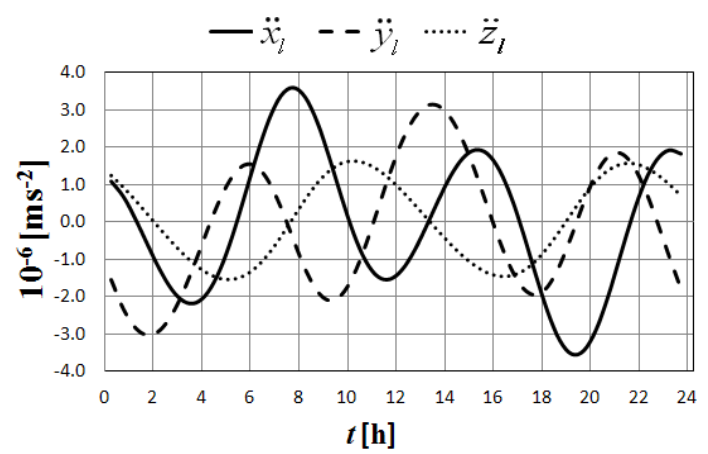

Fig. 7a. Acceleration components of the satellite GLN 15 due to lunar gravitation (2015-01-13).

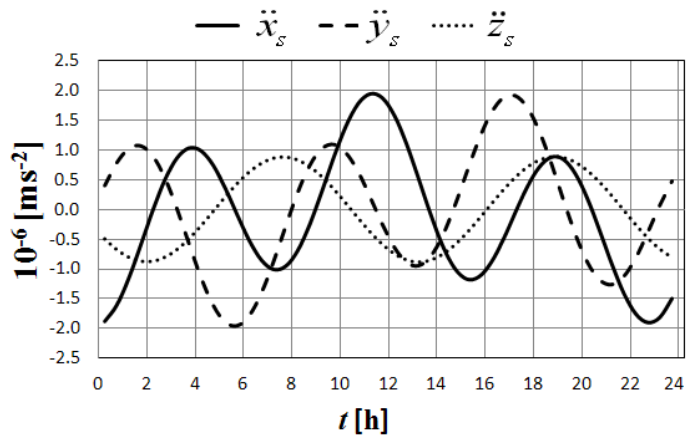

Fig. 7b. Acceleration components of the satellite GLN 15 due to solar gravitation (2015-01-13).

In Fig. 8 are presented comparisons of the calculated total lunar and solar accelerations $(l s)$ from broadcast (lsb) one of the GLN 15 (PZ-90.02 reference frame, 2015-01-13). One can see that some broadcast acceleration components are constants during several epochs.
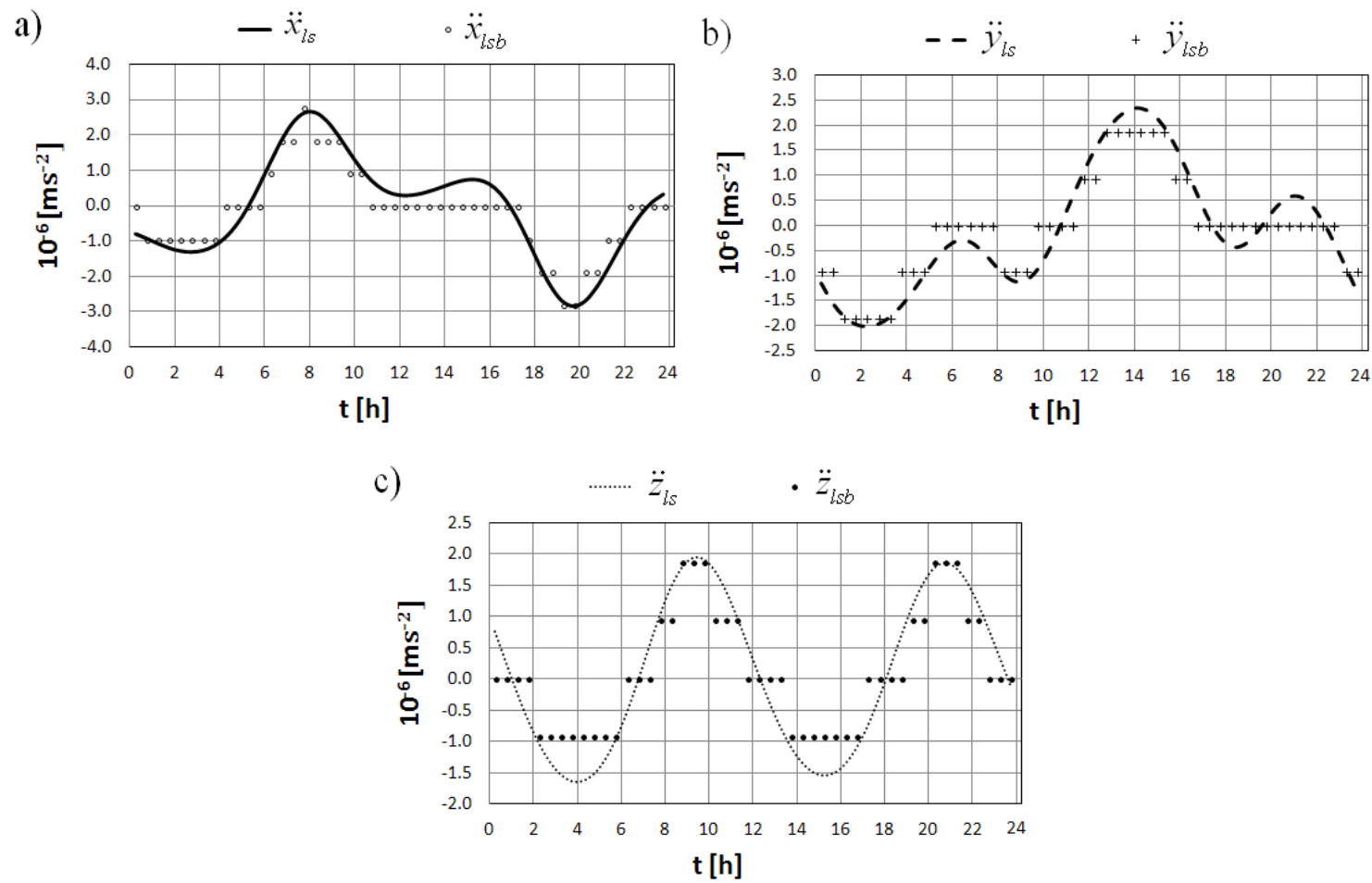

Fig. 8. The calculated lunar and solar accelerations $l s$ and broadcast $l s b$ one (in the PZ-90.02 reference frame) of the satellite GLN 15 (2015-01-13):

a) for $x$ component , b) for $y$ component, c) for $z$ component.

\section{NUMERICAL EXAMPLES}

First, on the base of broadcast ephemerides the intermediate Eulerian orbital elements of the satellite GLN 15 are computed for 48 epochs between $0^{\mathrm{h}} 15^{\mathrm{m}}$ to $23^{\mathrm{h}} 45^{\mathrm{m}}$ UTC (2015-01-13) at 30 minutes interval. Based on these orbital elements at epoch $t_{i}$, forward (for 30 minutes) at epoch $t_{i+1}=t_{i}+30^{\mathrm{m}}$ positions and velocities of the GLN 15 satellite are computed. The total lunar-solar accelerations were calculated according to the formulae given in GLONASS ICD 
2008. The differences $\left(x_{b}-x_{p}, y_{b}-y_{p}, z_{b}-z_{p}\right)$ between position components, obtained from broadcast ephemerides (subscript $\mathrm{b}$ ) at epoch $t_{i+1}$ and from predicted position components (subscript p) calculated on the base of Eulerian elements at epoch $t_{i}$ are given in Fig. 9. The

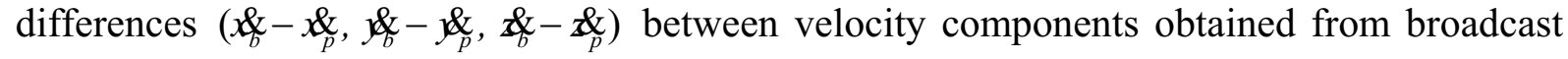
ephemerides at epoch $t_{\mathrm{i}+1}$ minutes and from predicted components calculated on the base of Eulerian elements at epoch $t_{i}$ are given in Fig. 10.

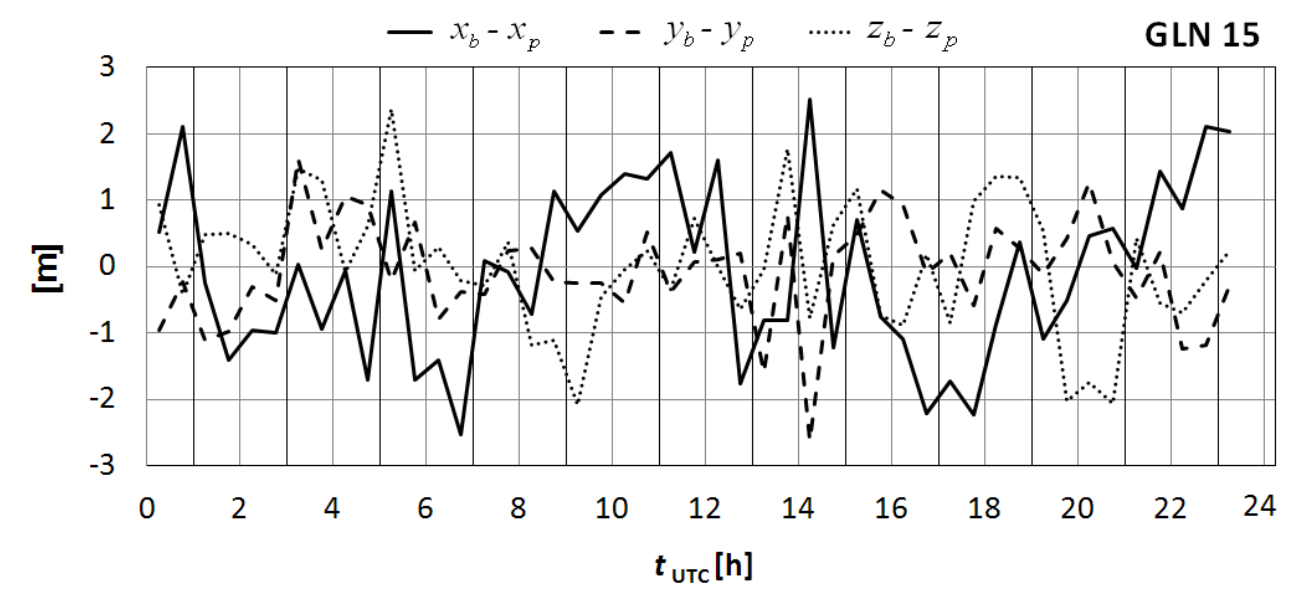

Fig. 9 Differences between the coordinates of the satellite GLN 15 given in navigation file and its values predicted forward $30^{\mathrm{m}}$, on 2015-01-13.

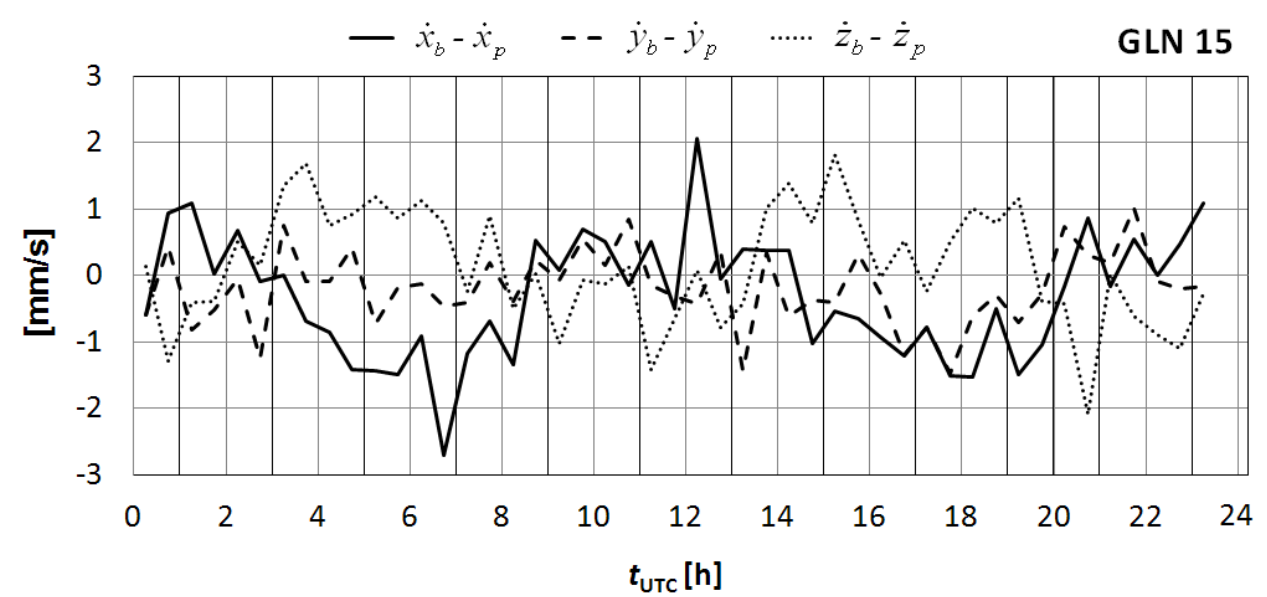

Fig. 10 Differences between the velocities of the satellite GLN 15 given in navigation file and its values predicted forward $30^{\mathrm{m}}$, on 2015-01-13.

In the next numerical example, on the base of known orbital elements of all (24) GLONASS satellites obtained at epoch $t_{i}$, forward for $30^{\mathrm{m}}\left(t_{i+1}\right)$ the positions and velocities are predicted, and then the results are compared with the corresponding coordinates given in the broadcast ephemerides at epoch $t_{i+1}$, according to the following formulas:

$$
\begin{aligned}
& d=\sqrt{\left(x_{b}-x_{p}\right)^{2}+\left(y_{b}-y_{p}\right)^{2}+\left(z_{b}-z_{p}\right)^{2}}, \\
& v=\sqrt{\left(x_{p}\right)^{2}+\left(x_{-}-y_{p}\right)^{2}+\left(z-z_{p}\right)^{2}} .
\end{aligned}
$$

The comparison was performed for two variants o calculations. In variant 1 (Fig 11a, 12a), the total lunar-solar accelerations were computed according to the formulae given in GLONASS ICD 2008. In variant 2 (Fig 11b, 12b), the broadcast lunar-solar accelerations were used. 


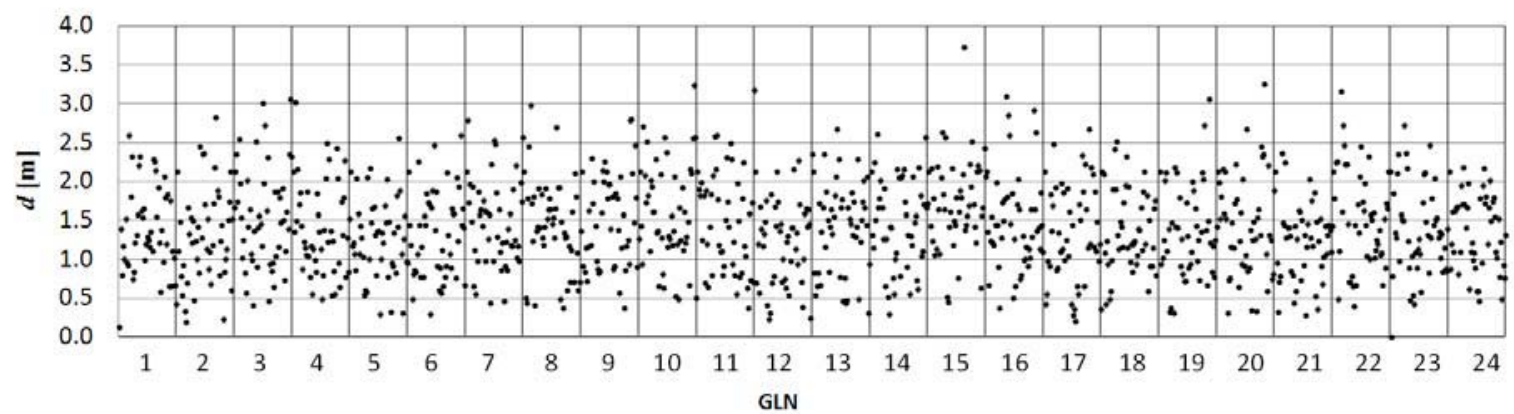

Fig. 11a Distances $d$ between broadcast and predicted satellite positions, obtained in the first variant of calculations, for all GLN satellites (2015-01-13).

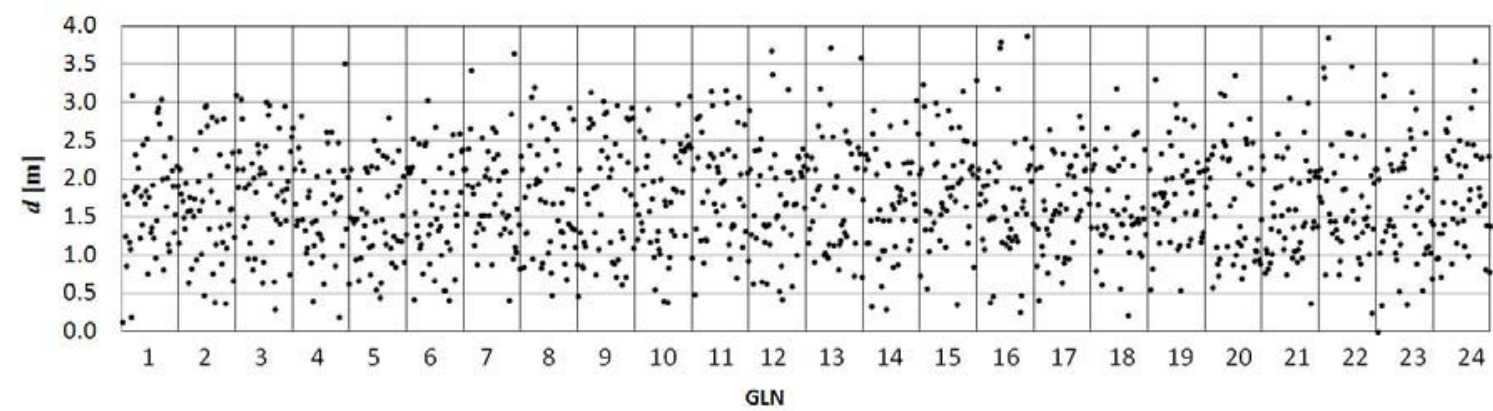

Fig. 11b Distances $d$ between broadcast and predicted satellite positions, obtained in the second variant of calculations, for all GLN satellites (2015-01-13).

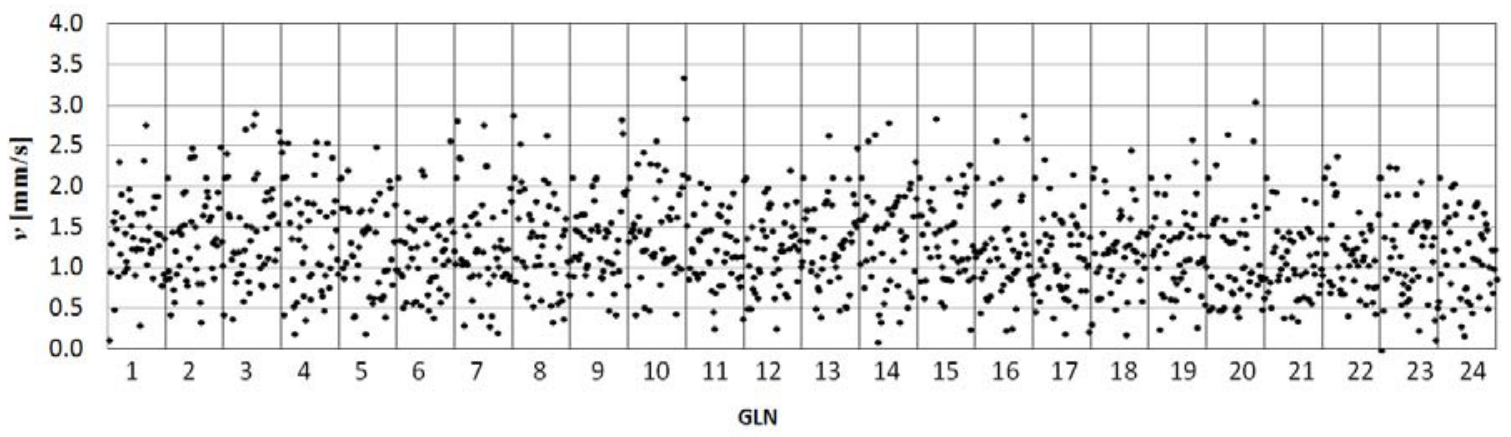

Fig. 12a Differences $v$ between broadcast and predicted satellite velocities, obtained in the first variant of calculations, for all GLN satellites (2015-01-13).

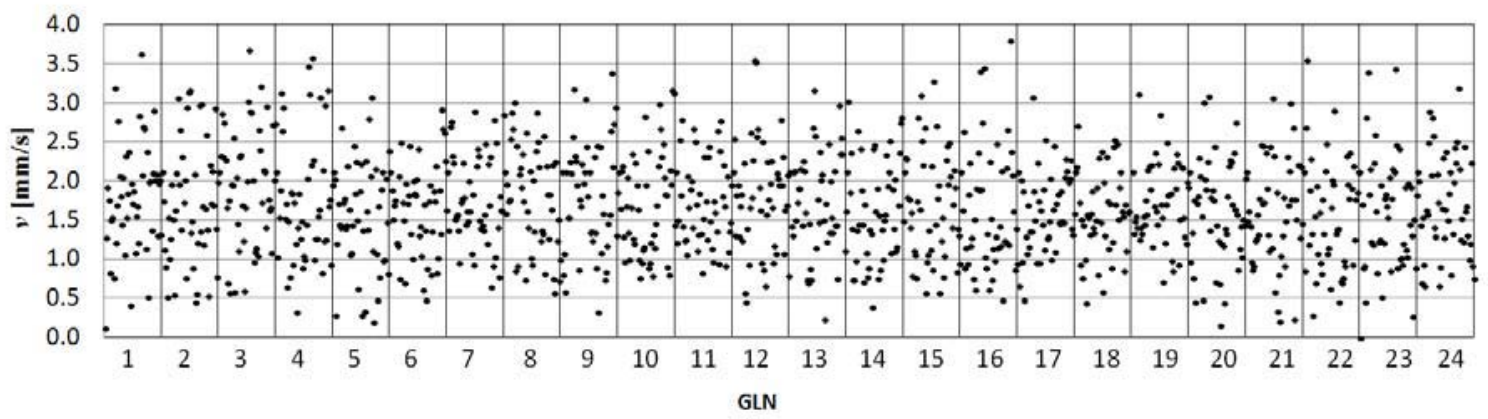

Fig. 12b Differences $v$ between broadcast and predicted satellite velocities, obtained in the second variant of calculations, for all GLN satellites (2015-01-13). 
Table 2 shows comparison of the results of the two variants of calculations.

Table 2. Average values of distances $d$ (eq. 5) and differences $v$ (eq. 6), and the corresponding standard deviations, obtained in two variants of calculations.

\begin{tabular}{|c|c|c|}
\hline $\begin{array}{c}\text { Variant of } \\
\text { calculations }\end{array}$ & 1 & 2 \\
\hline $\bar{d}[\mathrm{~m}]$ & 1.4 & 1.8 \\
\hline$\sigma_{d}[\mathrm{~m}]$ & 0.6 & 0.7 \\
\hline $\bar{v}[\mathrm{~mm} / \mathrm{s}]$ & 1.3 & 1.7 \\
\hline$\sigma_{v}[\mathrm{~mm} / \mathrm{s}]$ & 0.6 & 0.7 \\
\hline
\end{tabular}

The results presented in Fig.: 9,.., 12b are more accurate than the mean square error of broadcast positions and velocities of GLONAS-M satellites, which is about $10 \mathrm{~m}$ and 2.3 $\mathrm{mm} / \mathrm{s}$, respectively (ICD-GLONASS, 2008).

\section{CONCLUSIONS}

As result of analyses it was stated that proposed analytical method computation position and velocity of GLONASS satellites is an interesting alternative for presently used numerical methods. The results presented in this paper show that the proposed analytical method can be successfully used in interpolation and prediction of the position of GLONASS satellites.

In summary this paper has presented the expansion of the ICD-GLONASS 2008 algorithm of computation of the acceleration components due to luni-solar perturbation.

At present, on the base of the generalized problem of two fixed centers the precise theories of motion of Earth artificial satellites and satellites of other planets were developed (Lukyanov et al. 2005), (Golikov, 2012). The same papers described the basic stages of the development of modern high-precision theories of the motion of planetary satellites that use the intermediate orbit from a generalized problem of two fixed centers.

The obtained results open up new prospects for practical applications of the generalized problem of two fixed centers. They can be used for analytical studies of the motion not only of GLONASS satellite but also GPS and Galileo.

Acknowledgements. This work has been partially supported by the project No. 11.11.150. 006, AGH University of Science and Technology in Krakow.

\section{REFERENCES}

Aksenov E.P., Grebenikov E.A., Demin V.G. (1963). The generalized problem of motion about two fixed centers and its application to the theory of artificial Earth satellites, Soviet Astron. - AJ, Vol. 7, No. 2, American Institute of Physics, 276-283.

Aksenov E.P. (1977). Theory of motion artificial Earth's satellites (in Russian), Nauka Press, Moscow, p.360. 
Demin V.G. (1970). Motion of an artificial satellite in an eccentric gravitation field, translated and published as NASA Technical translation, TT F-579, Wshington D.C. (Translation from Dvizheniye Iskusstvennogo Sputnika $v$ Netsentral'nom Pole Tyagoteniya, Nauka Press, Moscow, 1968).

GLONASS Interface Control Document (ICD), Edition 5.1, Moscow, 2008.

Golikov A. R. (2012). THEONA - a numerical-analytical theory of motion of artificial satellites of celestial bodies, Cosmic Research, Vol. 50, No. 6, 449-458.

Góral W., Skorupa B. (2012). Determination of intermediate orbit and position of GLONASS satellites based on the generalized problem of two fixed centers, Acta Geodynamica et Geomaterialia, Vol. 9, No. 3 (167), 283-290.

Lukyanov L. G., Emelyanov N. V., Shirmin G. I. (2005). Generalized problem of two fixed centers or the Darboux-Gredeaks problem, Cosmic Research, Vol. 43, No. 3, 186-191.

Van Flandern T., Pulkkinen K. (1979). Low precision formulae for planetary positions, Astrophysical Journal Supplement Series, 41, 391-411.

Received: 2014-07-08,

Reviewed: 2015-06-01, by Z. Rzepecka,

Accepted: 2015-07-03. 